\title{
Increased phosphorylated p38 mitogen- activated protein kinase in COPD lungs
}

\author{
Kate Gaffey ${ }^{1,2}$, Sophie Reynolds ${ }^{1,2}$, Jonathan Plumb 1 , Manminder Kaur ${ }^{1}$ and \\ Dave Singh ${ }^{1}$ \\ Affiliations: \\ 'University of Manchester, NIHR Translational Research Facility, University Hospital of South Manchester, \\ Manchester, UK. \\ ${ }^{2}$ Contributed equally to this work and are joint first authors.
}

\section{Correspondence:}

Jonathan Plumb, University of Manchester, NIHR Translational Research Facility, Manchester Academic Health Science Centre, University Hospital Of South Manchester Foundation Trust, Southmoor Road, Manchester M23 9LT, UK.

E-mail: jonathan.plumbamanchester.ac.uk

ABSTRACT The p38 mitogen-activated protein kinase (MAPK) pathway is upregulated in chronic obstructive pulmonary disease (COPD). To date, dual labelling to identify cell-type-specific presence of phosphorylated (phospho-)p38 MAPK has not been carried out.

Phospho-p38 MAPK was quantified in a variety of cell types in the lung tissue of 20 COPD patients, 12 smokers and 12 nonsmokers using immunohistochemistry. Paired blood and sputum neutrophils (from seven subjects with COPD), and CD8 and epithelial cells (from three subjects with COPD) were cultured with a p38 MAPK inhibitor. Supernatant tumour necrosis factor- $\alpha$ and CXCL8 levels were analysed by ELISA. Sputum and blood neutrophil cytospins were analysed for phospho-p38 MAPK.

Phospho-p38 MAPK was increased in bronchial epithelial cells, macrophages and CD20+ and CD8+ lymphocytes in COPD lungs. Sputum and lung tissue neutrophils were devoid of phospho-p38 in all patient groups. The p38 MAPK inhibitor SB100 attenuated pro-inflammatory mediator release in COPD lung CD8 cells and airway epithelia, but there was no effect on COPD sputum neutrophils.

Our data indicate cell-specific anti-inflammatory effects of p38 MAPK inhibition in the lung.

@ERSpublications

p38 MAPK inhibition causes cell-specific anti-inflammatory effects in the lung

http://ow.ly/lbYX8

This article has supplementary material available from www.erj.ersjournals.com

Received: Oct 032011 | Accepted after revision: Sept 272012 | First published online: Oct 112012

Conflict of interest: Disclosures can be found alongside the online version of this article at www.erj.ersjournals.com

Copyright @ERS 2013 


\section{Introduction}

Chronic obstructive pulmonary disease (COPD) is characterised by poorly reversible airflow obstruction. There is also evidence of progressive airway inflammation [1]. Glucocorticoids are the most commonly used anti-inflammatory drug in COPD, but have limited effects on airway inflammation and disease progression $[2,3]$. Novel therapeutic approaches targeting inflammation in COPD are needed.

The p38 mitogen-activated protein kinase (MAPK) intracellular signalling pathway is activated by a variety of extracellular stimuli, including pro-inflammatory cytokines and Toll-like receptor (TLR) agonists $[4,5]$. p38 MAPK activation causes histone modifications within the promoter regions of a subset of genes; this increases accessibility for transcription factors such as nuclear factor $\mathrm{\kappa B}$ to these regions enhancing inflammatory gene expression [6]. Additionally, p38 MAPK acts post-transcriptionally by stabilising mRNAs, and promotes protein translation [7]. p38 MAPK inhibitors reduce cytokine production from alveolar macrophages [8-11] and are in clinical development for the treatment of COPD [12, 13].

There are increased numbers of inflammatory cells in the lungs of COPD patients, including lymphocytes, macrophages and neutrophils [1]. COPD patients also have increased numbers of pulmonary lymphoid follicles [1], which may function as antigen-presenting sites that promote auto-immune processes [14]. RENDA et al. [15] used single-label immunohistochemistry to demonstrate increased expression of activated p38 MAPK in the alveolar macrophages of COPD patients compared with controls. The specific expression of activated p38 MAPK on other relevant lung cells, such as epithelial cells, lymphocytes and neutrophils, has not been described. Furthermore, although the anti-inflammatory effects of p38 MAPK inhibitors on cytokine production from COPD alveolar macrophages is well documented [8-11], there is little data on the effects of this class of drug on other relevant immune cell types within the lungs of COPD patients.

There are four isoforms of p38 MAPK, which are encoded by separate genes; $38 \alpha, \mathrm{p} 38 \beta, \mathrm{p} 38 \delta$ and p38 $\gamma$. The expression of these isoforms varies between tissues and cell types [16]. The p38 $\alpha$ and p38 $\beta$ isoforms play predominant roles in immune cell activation, so the majority of p38 MAPK inhibitors developed for the treatment of inflammation have been targeted against these isoforms in order to avoid unwanted physiological effects through p38 $\gamma$ and $\mathrm{p} 38 \delta$ inhibition. It has been shown in glomerulonephritis that p38 $\alpha$ is the most highly expressed isoform in infiltrating leukocytes in the kidney [17], but there was also evidence of $\mathrm{p} 38 \beta$ and $\mathrm{p} 38 \gamma$ isoform expression in structural cell types. The expression levels of $\mathrm{p} 38$ MAPK isoforms in the lungs of COPD patients have not been quantitatively studied; this would identify the isoforms relevant to the pathophysiology of COPD.

The aims of the current study were as follows: 1) to characterise the cell-specific expression of activated p38 MAPK in COPD lungs compared with controls by using dual labelled immunofluorescence; 2) to investigate the anti-inflammatory effects of p38 MAPK inhibition on cytokine production from neutrophils, epithelial cells and lymphocytes isolated from COPD lungs; and 3) to investigate p38 MAPK isoform expression in lung tissue from COPD patients compared with controls.

\section{Methods}

Study subjects

Patients undergoing surgical resection for suspected or confirmed lung cancer were recruited. Patients with a previous diagnosis of COPD according to the Global Initiative for Chronic Obstructive Lung Disease guidelines [18] with at least a 10 cigarette pack-year history and airflow obstruction defined as forced expiratory volume in $1 \mathrm{~s}\left(\mathrm{FEV}_{1}\right)<80 \%$ and $\mathrm{FEV} 1 /$ forced vital capacity (FVC) $<70 \%$ were recruited. Controls consisted of smokers with $>10$ pack-year smoking history and normal pulmonary function and lifelong nonsmokers. Some controls had evidence of FEV1/FVC $<70 \%$ due to obstruction by tumour. In a separate study, COPD patients, smoking and nonsmoking for sputum induction, and COPD patients for isolated neutrophil work, were recruited. Additionally, three COPD patients were recruited for bronchoscopy for the isolation of airway epithelial cells. The demographics are shown in table 1. All patients gave written informed consent. The study was approved by the local ethics committee.

\section{Analysis of p38 mRNA levels}

Levels of $\mathrm{p} 38 \alpha, \mathrm{p} 38 \beta, \mathrm{p} 38 \delta$ and p38 $\gamma$ were measured by qPCR in COPD, smoking and nonsmoking patients. Levels of expression were normalised to GAPDH for analysis (described in the online supplementary material).

\section{Immunohistochemistry}

Tissue blocks were obtained from an area of the lung as far distal to the tumour as possible, and processed as described previously [19]. Blocks were labelled using anti-phospho-p38 MAPK primary antibody. Dual label immunofluorescence with phospho-p38 was performed with one of the following primary antibodies: 
neutrophil elastase, CD20, CD8 or CD4. Further details of methods and antibodies are described in the online supplementary material.

\section{Image analysis}

The percentage of phospho-p38+ CD20+, CD8+ and CD4+ cells within inflammatory follicles and within the subepithelium was quantified. Total numbers of phospho-p38+ neutrophils, small airway epithelial cells and macrophages (identified by morphology) were also quantified. For dual-label images, fluorescent images from the same field were captured and digitally merged to determine the phospho-p38 positive cells. Digital micrographs were obtained through the use of a Nikon Eclipse 80i microscope (Nikon UK Ltd, Kingston upon Thames, UK) equipped with a QImaging digital camera (Media Cybernetics, Marlow, UK) and ImagePro Plus 5.1 software (Media Cybernetics). Cell counts, follicle area and epithelial and subepithelial length were quantified using the ImagePro Plus 5.1 software. Cell counts were standardised to the number of positive cells per $\mathrm{mm}^{2}$ of the area of interest.

\section{Cell culture}

Neutrophils

Isolated blood and sputum neutrophils (cell isolation details are provided in the online supplementary material; the sputum cell counts are shown in table 2) were pre-treated with a p38 $\alpha$ and p38 $\beta$ MAPK selective inhibitor SB100 (GlaxoSmithKline, Stevenage, UK) using final concentrations of 10-1000 nM before the addition of lipopolysaccharide (LPS) $\left(100 \mathrm{ng} \cdot \mathrm{mL}^{-1}\right.$ ) (Sigma Aldrich, Poole, UK) for $24 \mathrm{~h}$. LPS $100 \mathrm{ng} \cdot \mathrm{mL}^{-1}$ is commonly used for neutrophil stimulation [20], and we confirmed that this is a suboptimal concentration for cytokine production (fig. S1a). Supernatants were removed and stored at $-80^{\circ} \mathrm{C}$ for cytokine analysis. Remaining cells were removed and centrifuged (Juan GR4i centrifuge; Thermo Fisher Scientific) at $400 \times g$ for $10 \mathrm{~min}$ at $4^{\circ} \mathrm{C}$. Cell viability and counts were determined before preparing cytospins and fixing in $4 \%$ paraformaldehyde prior to immunocytochemical analysis (see online supplementary material for methods). In order to determine cell viability, trypan blue exclusion was assessed before and after SB100 (1000 nM) treatment. To determine the extent of apoptosis before and after SB100 (1000 nM) treatment, DNA fragmentation and presence of condensed nuclei were assessed by terminal deoxynucleotidyl transferase dUTP nick end labeling (TUNEL) and nuclear morphology respectively (described in online supplementary material). Apoptosis was determined by examining the disappearance of chromatin bridges between nuclear lobes (early apoptosis) and shrinkage or fragmentation of the nucleus (late apoptosis). The percentage of neutrophils appearing normal, as well as early and late apoptotic neutrophils, was assessed by counting a total of 300 neutrophils.

\section{CD8 cells}

Isolated pulmonary CD8 cells (the isolation protocol is detailed in the online supplementary material) were seeded at $5 \times 10^{4}$ cells per well before pre-treatment with SB100 $(0.1-1000 \mathrm{nM})$. Cells were then stimulated

\section{TABLE 1 Subject demography}

\begin{tabular}{|c|c|c|c|c|c|c|c|c|}
\hline \multicolumn{3}{|c|}{ Lung tissue (PCR and IHC) } & \multicolumn{3}{|c|}{ Sputum cells (ICC) } & \multicolumn{3}{|c|}{ In vitro cell culture } \\
\hline COPD & Smokers & Nonsmokers & COPD & Smokers & Nonsmokers & $\begin{array}{l}\text { CD8 } \\
\text { COPD }\end{array}$ & $\begin{array}{l}\text { Epithelial } \\
\text { COPD }\end{array}$ & $\begin{array}{l}\text { Neutrophils } \\
\text { COPD }\end{array}$ \\
\hline
\end{tabular}

\begin{tabular}{|c|c|c|c|c|c|c|c|c|c|}
\hline Subjects & 31 & 19 & 21 & 8 & 6 & 4 & 3 & 3 & 7 \\
\hline Male/female & $17 / 14$ & $8 / 11$ & $8 / 13$ & $5 / 3$ & $0 / 6$ & $3 / 1$ & $2 / 1$ & $3 / 0$ & $4 / 3$ \\
\hline Age years & $66.4 \pm 7.2$ & $61.5 \pm 13.1$ & $59 \pm 15.6$ & $72.5 \pm 4.4$ & $52.3 \pm 6.8$ & $54 \pm 14.7$ & $63 \pm 3.6$ & $60 \pm 6.9$ & $74.1 \pm 3.1$ \\
\hline $\begin{array}{l}\text { Smoking history } \\
\text { pack-years }\end{array}$ & $50.9 \pm 25.6$ & $44.3 \pm 29.4$ & 0 & $52 \pm 8.9$ & $29.8 \pm 8.6$ & 0 & $61.3 \pm 38.7$ & $21 \pm 1.3$ & $47.6 \pm 6.9$ \\
\hline FEV 1 L & $1.8 \pm 0.5$ & $2.3 \pm 0.73$ & $2.2 \pm 0.8$ & $1.0 \pm 0.3$ & $2.8 \pm 0.6$ & $3.2 \pm 1.2$ & $1.6 \pm 0.2$ & $1.8 \pm 0.4$ & $1.1 \pm 0.3$ \\
\hline FEV $1 \%$ pred & $66.7 \pm 11.6$ & $91.3 \pm 16.7$ & $93.6 \pm 19$ & $43.6 \pm 10.8$ & $101.0 \pm 9.1$ & $106.4 \pm 9.6$ & $59.7 \pm 14.6$ & $53.5 \pm 6.2$ & $47.0 \pm 15.7$ \\
\hline FEV1/FVC \% & $57.2 \pm 8.6$ & $73.8 \pm 7.2$ & $75.7 \pm 10.5$ & $42.0 \pm 10.3$ & $76.2 \pm 4.2$ & $75.0 \pm 3.6$ & $52 \pm 12.2$ & $50 \pm 8.7$ & $46.7 \pm 8.7$ \\
\hline
\end{tabular}

Data are presented as $\mathrm{n}$ or mean \pm SD. IHC: immunohistochemistry; ICC: immunocytochemistry; COPD: chronic obstructive pulmonary disease; FEV1: forced expiratory volume in $1 \mathrm{~s} ; \%$ pred: \% predicted; FVC: forced vital capacity; ICS: inhaled corticosteroid. ${ }^{\#}$ : neutrophils were isolated from both sputum and blood. 
TABLE 2 Sputum differential cell counts from samples used in cell culture experiments

Before enrichment

Total cell count cells $\times 10^{6}$
Total neutrophil count cells $\times 10^{6}$
Neutrophil $\%$
Total macrophage count cells $\times 10^{6}$
Macrophage $\%$
Eosinophil $\%$
Lymphocyte $\%$
Squamous cell \%

$14.2 \pm 13.5$
$10.3 \pm 9.7$
$73.5 \pm 4.6$
$3.3 \pm 3.5$
$22.5 \pm 4.4$
$1.0(0.5-1.8)$
$0.3(0-1.8)$
$1(0-1.75)$

After enrichment
$8.84 \pm 8.9$
$8.0 \pm 8.2$
$89.5 \pm 3.1$
$0.7 \pm 0.6$
$9.0 \pm 3.6$
$0(0-1.75)$
$0(0-0.5)$
$0.25(0-3.75)$

Data are presented as mean \pm SD or median (range). Differential cell counts for sputum samples used for cell culture pre- and post-neutrophil isolation step $(n=7)$. Cell numbers are normalised per gramme of sputum.

for $24 \mathrm{~h}$ with interleukin (IL)-12 (10 ng $\cdot \mathrm{mL}^{-1}$; Peprotech, London, UK) and IL-18 (10 ng $\cdot \mathrm{mL}^{-1}$; Peprotech) for $24 \mathrm{~h}$ before harvesting supernatants for the measurement of interferon (IFN)- $\gamma$ by ELISA (eBioscience, Hatfield, UK). These cytokine concentrations were determined by preliminary concentration-response experiments in isolated blood CD8 cells; suboptimal IFN- $\gamma$ production coupled with p38 phosphorylation was observed (fig. S1b and c).

\section{Epithelial cells}

The isolation of epithelial cells is described in the online supplementary material. Epithelial cells were cultured in 96-well plates until 80\% confluent. Cells were pre-treated with SB100 $(1 \mu \mathrm{M})$ for $1 \mathrm{~h}$ before culturing with polyinosinic:polycytidylic acid (poly I:C) $\left(10 \mu \mathrm{g} \cdot \mathrm{mL}^{-1}\right.$; Invivogen, San Diego, CA, USA) for 24 h. Supernatants were harvested for the measurement of chemokine (C-C motif) ligand 5 (CCL5), CXCL8 and IL-6 by ELISA (R\&D Systems, Abingdon, UK).

\section{Cytokine measurements}

Supernatant levels of tumour necrosis factor (TNF)- $\alpha$, CXCL8, IFN- $\gamma$, IL-6 and CCL5 were determined using ELISA according to the manufacturer's instructions. The lower limit of detection for TNF- $\alpha$, CXCL8, IL-6 and CCL5 were $15.6 \mathrm{pg} \cdot \mathrm{mL}^{-1}, 32.5 \mathrm{pg} \cdot \mathrm{mL}^{-1} 9.375 \mathrm{pg} \cdot \mathrm{mL}^{-1}$ and $15.6 \mathrm{pg} \cdot \mathrm{mL}^{-1}$, respectively (R\&D Systems). The lower limit of detection of IFN- $\gamma$ was $7.8 \mathrm{pg} \cdot \mathrm{mL}^{-1}$ (eBioscience, Hatfield, UK).

\section{Statistical analysis}

Normality was assessed using the Kolmogorov-Smirnov test. Comparisons between COPD patients, smokers and nonsmokers were performed using one-way ANOVA followed by Bonferroni's post-test for parametrically distributed immunohistochemistry data. PCR data were analysed by nonparametric ANOVA (Kruskal-Wallis test) for between group comparisons and repeated measures ANOVA (Friedman test) for within group analyses, followed by Dunn's post-test. Paired t-tests were used to compare the effect of SB100 in cell cultures. Analysis was carried out using GraphPad InStat software version 3.06 (GraphPad Software, Inc., San Diego, CA, USA).

\section{Results}

p38 isoform expression in lung tissue

Gene expression levels of $\mathrm{p} 38 \alpha, \beta, \gamma$ and $\delta$ were analysed by qPCR in RNA extracted from COPD $(\mathrm{n}=11)$, smoking $(n=7)$ and nonsmoking $(n=10)$ lung tissue (fig. 1$)$. The $\alpha$ and $\delta$ isoforms were significantly increased in COPD lung tissue compared with nonsmoking $(\mathrm{p}<0.01$ and $\mathrm{p}<0.05$, respectively), while $\mathrm{p} 38 \alpha$ expression was also significantly increased in smokers compared with nonsmokers $(\mathrm{p}<0.01)$.

p $38 \alpha$ expression was increased compared with $\mathrm{p} 38 \beta$ and $\mathrm{p} 38 \gamma$ in COPD patients $(\mathrm{p}<0.01$ for both isoforms), and $\mathrm{p} 38 \alpha$ expression was also increased compared with $\mathrm{p} 38 \beta$ in smokers $(\mathrm{p}<0.01)$. There was no difference between the expression levels of the isoforms in nonsmokers.

\section{Phospho-p38 MAPK in lymphocytes}

Follicles

Dual label immunofluorescence showed that phospho-p38 MAPK was present in all the follicles analysed. 


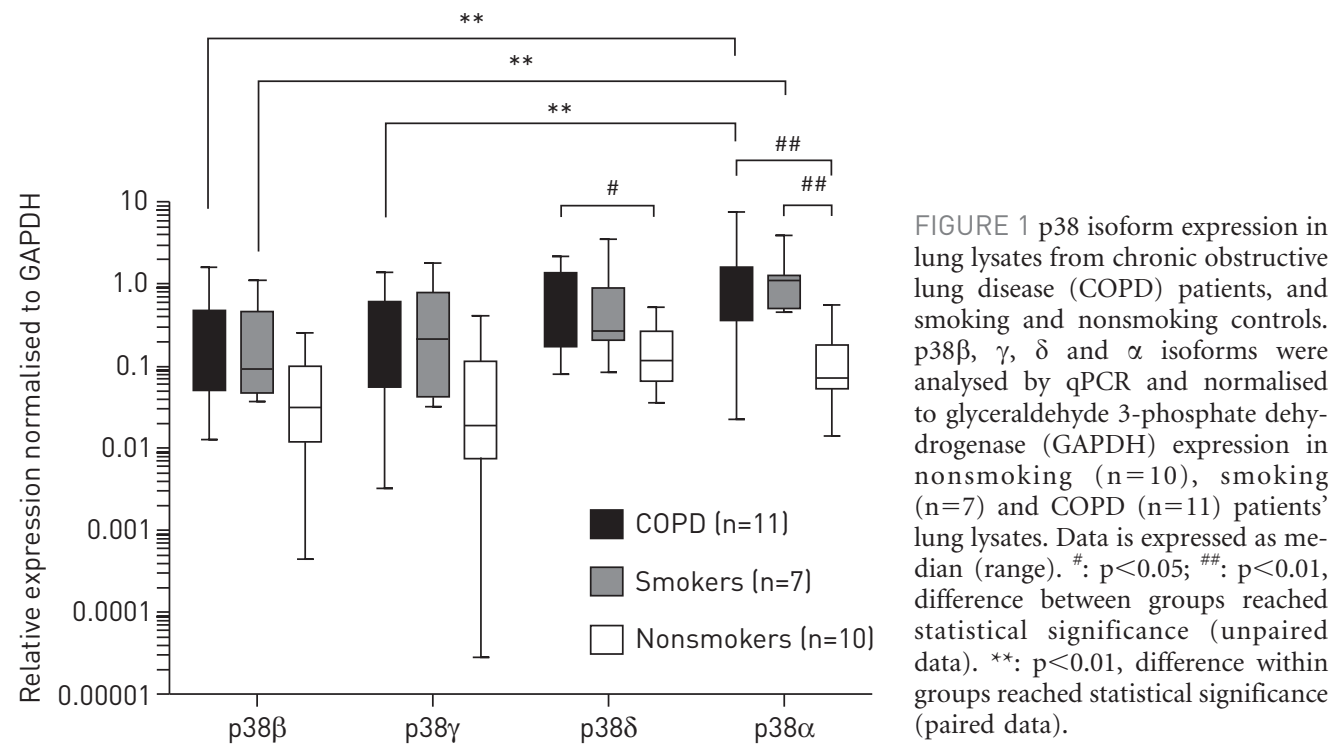

B-cells

Bonferroni's multiple comparisons tests showed that the percentage of CD20+ phospho-p38+ cells was significantly higher in COPD patients compared with smokers and nonsmokers $(\mathrm{p}<0.001$ for both comparisons; mean $86.6 \%, 44.1 \%$ and $30.9 \%$, respectively). Numbers of CD20+ phospho-p38+ were also significantly greater in smokers compared with nonsmokers $(\mathrm{p}<0.05)$ (figs $2 \mathrm{a}$ and 3 ).

\section{CD8 cells}

Bonferroni's multiple comparisons tests showed that the percentage of CD8+ phospho-p38+cells was significantly higher in COPD patients compared with nonsmokers $(\mathrm{p}<0.001)$ (mean $56.2 \%$ and $31.5 \%$, respectively). There was also a numerical trend towards increased numbers of CD8+ phospho-p38+ cells in COPD compared with smokers (mean 43.6\%) and in smokers compared with nonsmokers (mean 31.5\%), although this difference was not statistically significant ( $\mathrm{p}>0.05$ for both comparisons) (figs $2 \mathrm{~b}$ and 4 ).

\section{CD4 cells}

The numbers of phospho-p38+ CD4+ cells within inflammatory follicles was much lower in all patient groups; typically $<20 \%$ of CD4+ cells were positive for phospho-p38 MAPK. Statistically, there were no differences between any of the groups (fig. 2c)

\section{Subepithelium}

The number of subepithelial lymphocytes and the number of subepithelial lymphocytes positive for phospho-p38 MAPK was similar across all three patient groups (table 3). Phospho-p38 MAPK was absent in subepithelial CD4+ lymphocytes. Numbers of CD20+and CD8+ phospho-p38+ cells were significantly lower compared with phospho-p38+ lymphocytes within follicles (ANOVA $p<0.0001$ for comparisons of both cell types).

\section{Phospho-p38 MAPK in macrophages}

Alveolar macrophages

The percentage of phospho-p38+ alveolar macrophages was significantly greater in COPD patients compared with both smokers and nonsmokers (mean 70.0\%, 56.4\% and 28.5\%, respectively, Bonferroni's multiple comparisons tests COPD versus smokers, $\mathrm{p}<0.01$; COPD versus nonsmokers, $\mathrm{p}<0.001$ ), and in smokers compared with nonsmokers (Bonferroni's multiple comparisons test $\mathrm{p}<0.001$ ) (figs $2 \mathrm{~d}$ and $5 \mathrm{a}-\mathrm{c}$ ).

\section{Sputum macrophages}

The percentage of phospho-p38+ sputum macrophages was significantly greater in COPD patients $(n=8)$ compared with both smokers $(n=6)$ and nonsmokers $(n=4)$ (mean 92.8\%, 59.3\% and 31.8\%, respectively; Bonferroni's multiple comparisons tests $\mathrm{p}<0.001$ for all comparisons) (figs $2 \mathrm{e}$ and $5 \mathrm{~d}-\mathrm{f}$ ). 

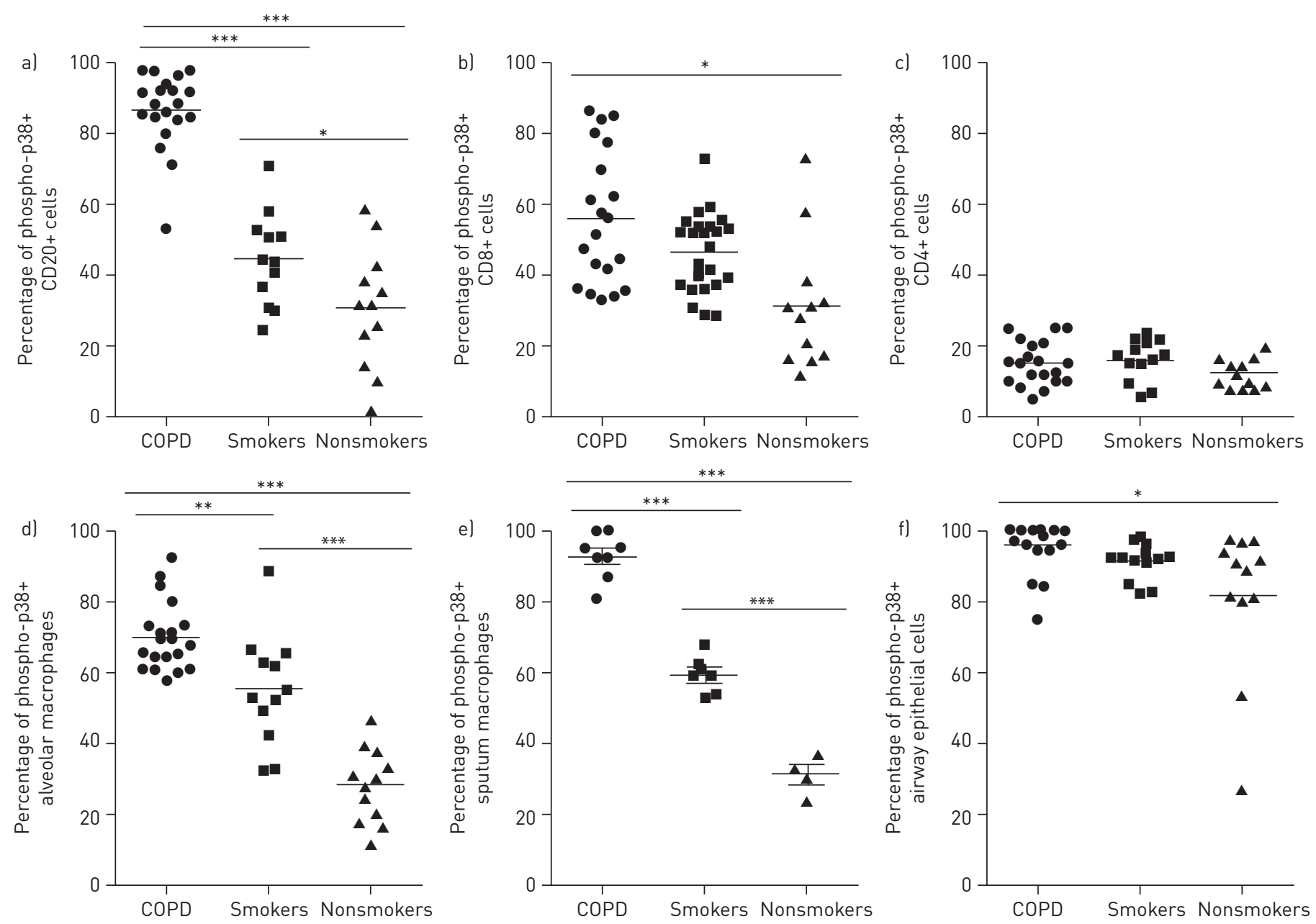

FIGURE 2 The mean percentage of phosphorylated (phospho-)p38 mitogen-activated protein kinase (MAPK)+ a) follicle CD20+ B-cells, b) follicle CD8+ cells, c) follicle CD4+ cells, d) lung tissue macrophages, e) sputum macrophages and f) small airway epithelial cells. COPD: chronic obstructive pulmonary disease. Differences between patient groups for each cell type: ${ }^{\star}: \mathrm{p}<0.05 ;{ }^{\star *}: \mathrm{p}<0.01 ;{ }^{* *}: \mathrm{p}<0.001$.

Phospho-p38 MAPK in epithelial cells

Phospho-p38 MAPK was present in the majority of small airway epithelial cells analysed in all three patient groups. Bonferroni's multiple comparisons tests showed that there was a significant increased percentage of phospho-p38+epithelial cells between COPD and nonsmokers (mean 96\% and 81\%, respectively; p $<0.05$ ). There was also a trend towards increased percentages of phospho-p38+ epithelial cells in COPD patients compared with smokers (mean 91\%), although this was not statistically significant (figs $2 \mathrm{f}$ and $5 \mathrm{~g}-\mathrm{i}$ ).

\section{Phospho-p38 MAPK in neutrophils}

Lung tissue neutrophils were devoid of phospho-p38 MAPK immunoreactivity in all patient groups (fig. 5j1). Sputum neutrophils also lacked phospho-p38 MAPK (fig. 5d-f). We examined phospho-p38 MAPK in neutrophils isolated from blood of COPD patients. Neutrophils examined immediately after isolation from blood did not have phospho-p38 MAPK, but phospho-p38 MAPK was induced following culture with LPS (fig. 6a and b). Although p38 MAPK expression was observed in sputum neutrophils (fig. S1d), phosphop38 MAPK was absent in sputum neutrophils cultured with LPS for $24 \mathrm{~h}$ (fig. $6 \mathrm{c}$ and d), indicating that the p38 MAPK pathway is not active in lung neutrophils.

\section{Cell specific effects of p38 MAPK inhibition}

\section{CD8 cells}

CD8 cells isolated from lung tissue of three COPD patients were pre-treated with SB100 before stimulating with IL-12 and IL-18 in combination. IFN- $\gamma$ production increased, from mean basal levels of $5.3 \mathrm{pg} \cdot \mathrm{mL}^{-1}$ to 

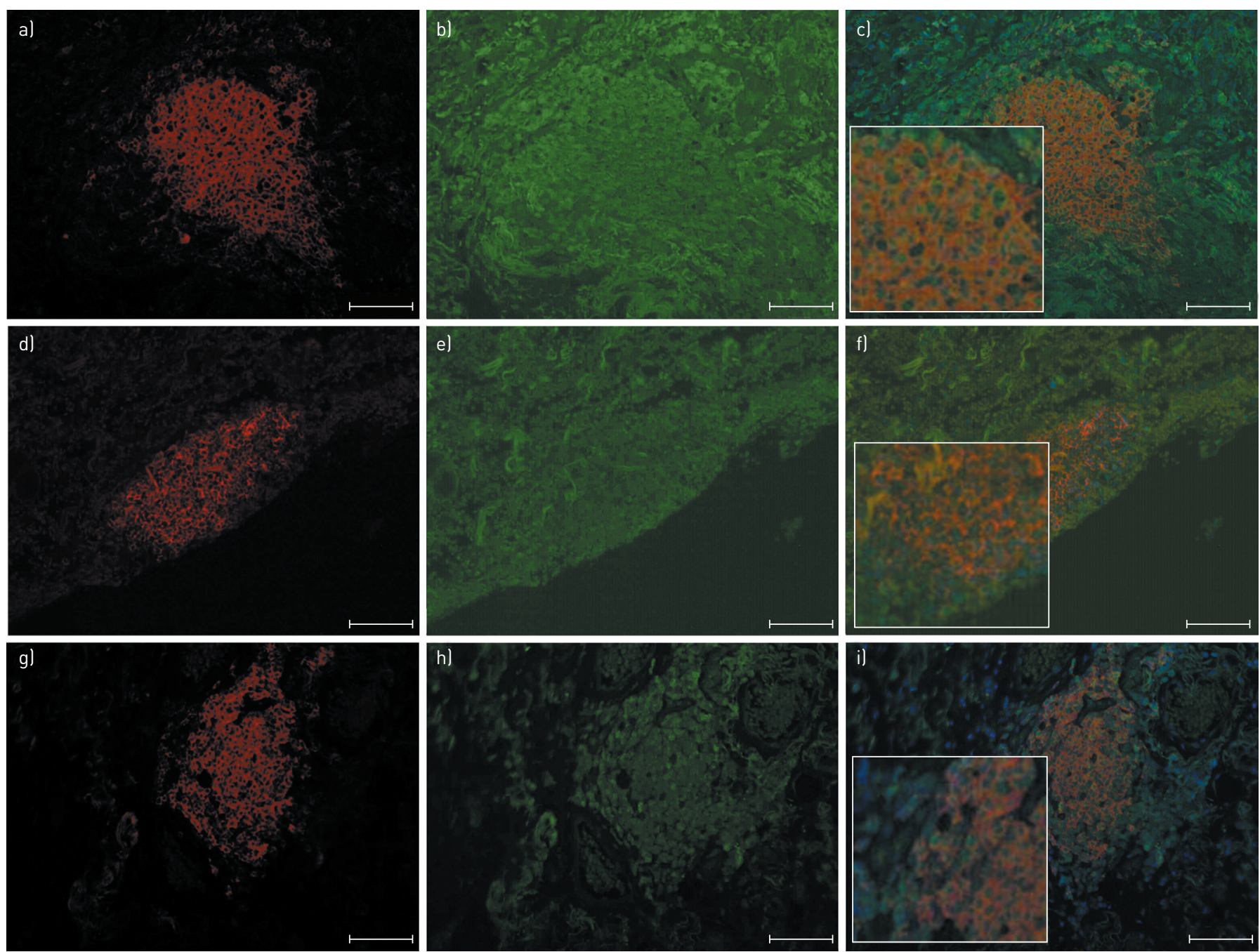

FIGURE 3 Representative images for the dual label immunofluorescent detection of phosphorylated (phospho-)p38 mitogen-activated protein kinase (MAPK) in $\mathrm{CD} 20+\mathrm{B}$-cells in inflammatory follicles within human lung tissue. Representative images from a-c) 20 chronic obstructive pulmonary disease patients, $\mathrm{d}-\mathrm{f}$ ) 12 smokers and g-i) 12 nonsmokers are shown. Cell nuclei were counterstained with 4',6-diamidino-2-phenylindole (blue). CD20+ cells were identified using an Alexa-488 conjugated goat anti-mouse secondary antibody (red; a, d and g) and phospho-p38 MAPK was detected using an Alexa 468-conjugated goat anti-rabbit secondary antibody (green; b, e and h). Composite images are shown (c, f and i). Green/yellow fluorescence is caused by intrinsically fluorescent tissue components, such as elastic fibres and red blood cells. Autofluorescence can be distinguished from positive fluorescence by forming a composite image of the red, green and blue channels. Autofluorescence is visible in all three channels and so appears as an amalgamation of the three colours. Positive fluorescence is visible in only one channel and thus appears as the pure colour. Magnification $\times 200$. Scale bars $=75 \mu \mathrm{m}$.

mean $1780 \mathrm{pg} \cdot \mathrm{mL}^{-1}$. SB100 inhibited IFN- $\gamma$ production in a dose-dependent manner, with maximal inhibition of $94.8 \%$ observed (fig. 7a).

\section{Epithelial cells}

Cells were pre-treated with SB100 (1000 nM) for $1 \mathrm{~h}$ prior to stimulating with poly I:C for $24 \mathrm{~h}$. Mean basal levels of IL-6 and CXCL8 were $85.5 \mathrm{pg} \cdot \mathrm{mL}^{-1}$ and $15258 \mathrm{pg} \cdot \mathrm{mL}^{-1}$ respectively. Basal levels of CCL5 were below the limit of detection. Poly I:C stimulation increased IL-6, CXCL8 and CCL5 release to $1580.1 \mathrm{pg} \cdot \mathrm{mL}^{-1}, 56335 \mathrm{pg} \cdot \mathrm{mL}^{-1}$ and $2643 \mathrm{pg} \cdot \mathrm{mL}^{-1}$, respectively. SB100 caused $50.7 \%, 38.7 \%$ and $26.7 \%$ inhibition of IL-6, CXCL8 and CCL5, respectively (fig. 7b).

\section{Neutrophils}

Isolated blood neutrophils (from seven COPD patients) were cultured for $24 \mathrm{~h}$ with and without LPS. The mean levels of unstimulated TNF- $\alpha$ and CXCL8 release were $527 \mathrm{pg} \cdot \mathrm{mL}^{-1}$ and $2857 \mathrm{pg} \cdot \mathrm{mL}^{-1}$, respectively, increasing to $2706 \mathrm{pg} \cdot \mathrm{mL}^{-1}$ and $7161 \mathrm{pg} \cdot \mathrm{mL}^{-1}$, respectively, after LPS stimulation. SB100 caused a dosedependent inhibition of cytokine production in both stimulated and unstimulated neutrophils (fig. $7 \mathrm{c}$ and d). There were no differences between percentage inhibition of TNF- $\alpha$ and CXCL8 in stimulated or unstimulated neutrophils. 

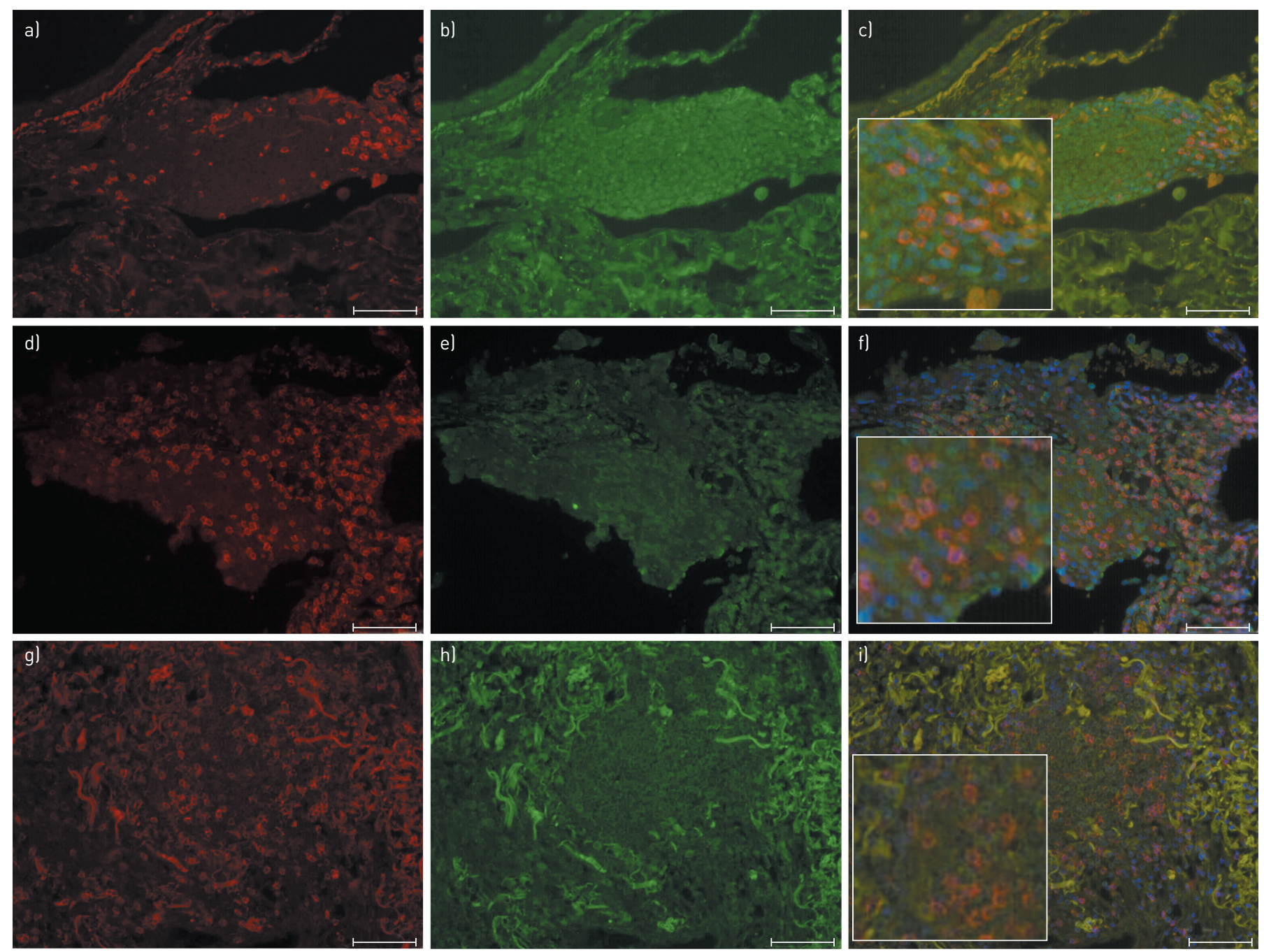

FIGURE 4 Representative images for the dual immunofluorescent detection of phosphorylated (phospho-)p38 mitogen-activated protein kinase (MAPK) in $\mathrm{CD} 8+$ cells within inflammatory follicles in lung tissue. Representative images from a-c) 20 chronic obstructive pulmonary disease patients, $\mathrm{d}-\mathrm{f}) 12 \mathrm{smokers}$ and g-i) 12 nonsmokers are shown. Cell nuclei were counterstained with $4^{\prime}, 6$-diamidino-2-phenylindole (blue). CD8+ cells were identified using an Alexa-488 conjugated goat anti-mouse secondary antibody (red; a, d and g) and phospho-p38 MAPK was detected using an Alexa 468 conjugated goat anti-rabbit secondary antibody (green; b, e and h). Composite images are also shown (c, f and i). Magnification $\times 200$. Scale bars $=75 \mu \mathrm{m}$.

Sputum samples from these seven COPD patients were also obtained. Previous studies have shown that LPS has no effect on cytokine production from isolated sputum neutrophils [21], which we also observed (data not shown). The mean levels of unstimulated TNF- $\alpha$ and CXCL8 release were $681 \mathrm{pg} \cdot \mathrm{mL}^{-1}$ and $4532 \mathrm{pg} \cdot \mathrm{mL}^{-1}$ in sputum neutrophils. In unstimulated neutrophils isolated from sputum, SB100 only inhibited TNF- $\alpha$ at the highest concentration $(1000 \mathrm{nM})$ and had no effect on CXCL8 production (fig. 7e and f). SB100 had a significantly lower effect on sputum neutrophils compared with LPS-stimulated and -unstimulated blood neutrophils at all concentrations for both TNF- $\alpha$ and CXCL8 $(p<0.05$ for comparisons of all concentrations using one-tailed paired t-tests).

Cell viability was assessed before and after SB100 $(1000 \mathrm{nM})$ treatment $(\mathrm{n}=4)$. There was no significant effect of SB100 on sputum neutrophil or blood neutrophil viability, measured by trypan blue exclusion, morphological analysis for apoptosis and TUNEL assay (figs S2 and S3).

\section{Discussion}

We have demonstrated increased phospho-p38 expression in specific cell types within the lungs of COPD patients compared with controls; the proportion of follicular B cells and CD8 lymphocytes, small airway bronchial epithelial cells and macrophages expressing immunoreactivity for phospho-p38 was increased in lung samples from COPD patients compared with controls. In these specific cell types, the overall pattern that we observed was for cigarette smoking to increase phospho-p38 MAPK, and the development of COPD 
TABLE 3 Mean number of CD20+, CD8+ and CD4+ lymphocytes per $\mathrm{mm}^{2}$ subepithelia and percentage of cell-specific phosphorylated p38 mitogen-activated protein kinase (MAPK)

\begin{tabular}{|c|c|c|c|c|c|c|c|c|}
\hline & \multicolumn{4}{|c|}{ Lymphocytes per $\mathrm{mm}^{2}$ subepithelia $\mathrm{n}$} & \multicolumn{4}{|c|}{ Phospho-p38 MAPK presence \% } \\
\hline CD20 & 181.64 & 113.47 & 108.81 & 0.55 & 4.35 & 4.84 & 3.24 & 0.90 \\
\hline CD8 & 438.82 & 400.31 & 380.93 & 0.62 & 15.43 & 8.91 & 3.93 & 0.86 \\
\hline CD4 & 84.44 & 60.36 & 57.4 & 0.98 & 0 & 0 & 0 & 0 \\
\hline
\end{tabular}

COPD: chronic obstructive pulmonary disease. ${ }^{\#}$ : 20 samples analysed; ${ }^{\circ}: 12$ samples analysed; ${ }^{+}$: for comparisons between patient groups for each cell type.

to cause a further increase. It has previously been reported that the proportion of alveolar macrophages expressing phospho-p38 MAPK is increased in COPD alveolar macrophages [15]; we have now identified other lung cell types that also show this pattern of increased phospho-p38 MAPK expression in COPD patients. p38 MAPK inhibition in isolated COPD lung CD8 cells and epithelial cells reduced cytokine production, demonstrating that the expression of phospho-p38 in these cells is associated with proinflammatory functions.

Lung neutrophils were devoid of phospho-p38 MAPK immunoreactivity in both COPD patients and controls. Additionally, p38 MAPK inhibition had no effect on cytokine production from COPD lung neutrophils; this suggests that p38 MAPK signalling does not play a role in the pro-inflammatory activity of COPD lung neutrophils. This contrasts with COPD blood neutrophils, where phospho-p38 was detected and was functionally involved in cytokine production. p38 MAPK inhibitors are currently being developed as anti-inflammatory drugs for COPD; it appears that these drugs can exert anti-inflammatory effects on certain lung cell types, such as B-cells, CD8 cells, macrophages and epithelial cells, but have no effect on lung neutrophils.

We observed that p38 $\alpha$ MAPK isoform gene expression levels were increased in lung tissue from COPD patients and smokers compared with nonsmokers, with no difference between COPD patients and smokers; this suggests that cigarette smoking upregulates p38 $\alpha$ gene expression. p38 $\alpha$ was also the most highly expressed isoform; this is the primary isoform target of the majority of p38 MAPK inhibitors. p $38 \delta$ expression was increased in COPD patients compared with nonsmokers, although there was no difference between COPD patients and smokers. p38 $\delta$ has previously been shown to be expressed in macrophages [9], and our results indicate upregulation of the expression of this isoform in the lungs of COPD patients. We also demonstrated expression of $\mathrm{p} 38 \gamma$ in COPD lung tissue at a similar level to control samples; this isoform appears to play a role in glucocorticoid resistant inflammation [22].

Increased numbers of lymphoid follicles in the small airways of COPD patients are associated with more severe disease [1]. We observed that the B-cell cores and CD8 cells within these follicles had increased phospho-p38 MAPK in COPD patients compared with controls. Interestingly, there was no increase in numbers of phospho-p38+ B-cells and CD8 cells within the subepithelial region of COPD patients compared with controls. This suggests that lymphocytes within the follicles have a different physiological function compared with other lung lymphocytes. This is perhaps not surprising as follicular lymphocytes lie within an environment that resembles lymph nodes rather than normal lung tissue, and function as important sites of antigen presentation [23, 24].

The numbers of follicular phospho-p38+ CD4+ cells were similar in all patient groups. The number of phospho-p38+CD4+ cells within these follicles was much lower than that observed for both CD8+ cells and CD20+ cells. Furthermore, phospho-p38 immunoreactivity was absent in CD4 cells in the subepithelium. This striking difference in phospho-p38 expression between different lymphocyte subtypes suggests that p38 MAPK signalling may not play a central role in the physiology of lung CD4 cells. However, caution should be applied to the interpretation of these immunohistochemistry data, which are taken from a snapshot in time, as it is possible that p38 MAPK signalling is activated in COPD CD4 cells at other times, for example, during exacerbations.

Previous studies have shown that pharmacological inhibition of p38 MAPK can reduce pro-inflammatory cytokine production from COPD macrophages [9-11]. We now show that p38 MAPK inhibition also reduces pro-inflammatory cytokine production from lung CD8 cells and epithelial cells from COPD 

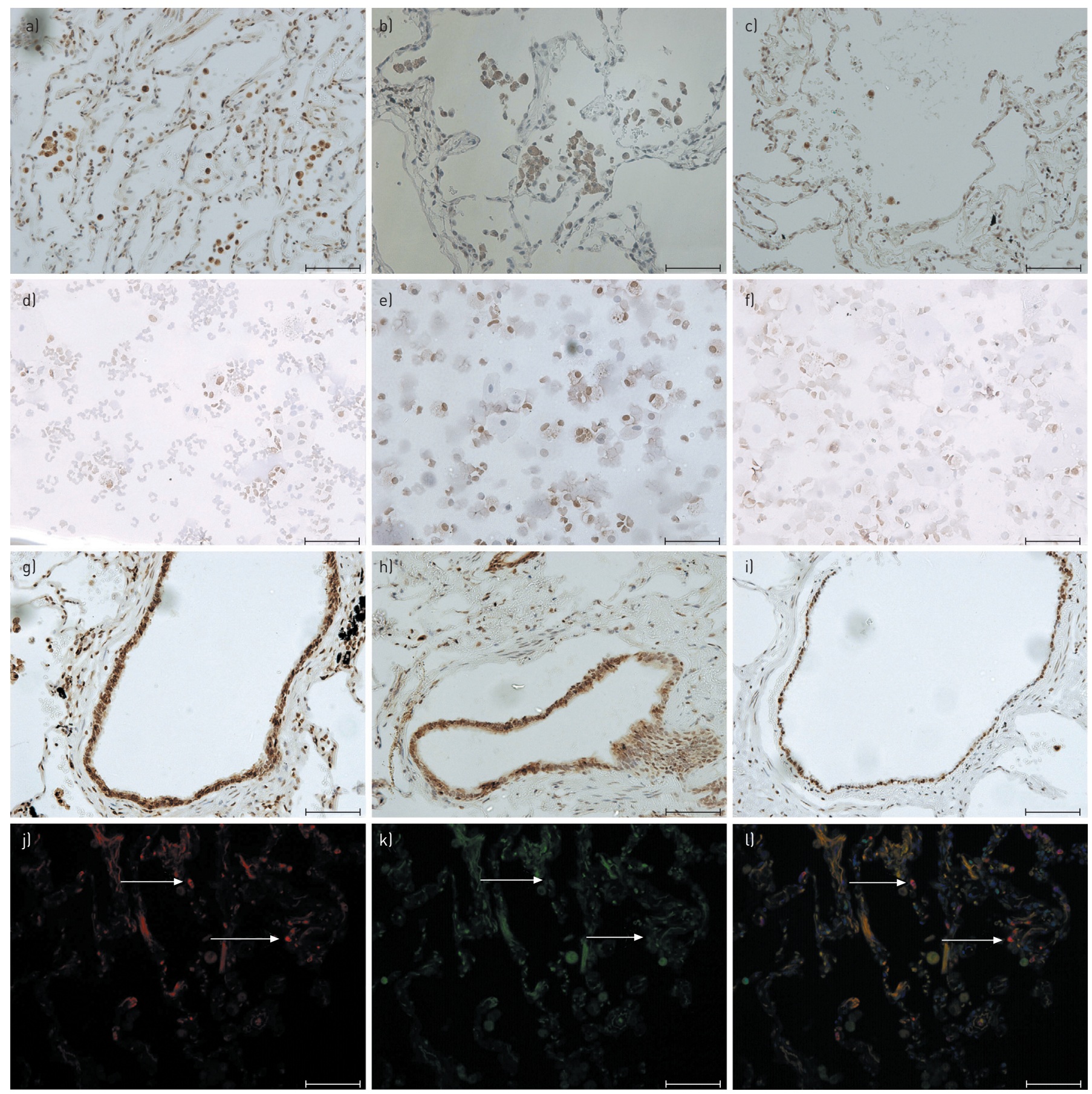

FIGURE 5 Representative images for the immunohistochemical and dual label immunofluorescent detection of phosphorylated (phospho-)p38 mitogenactivated protein kinase (MAPK) in human lung tissue and sputum cytospins. Representative images shown for (a-i) immunocytochemical and ( $\mathrm{j}-\mathrm{l}$ ) immunofluorescence tissue analysis from $(a, g$ and $j: n=20 ; d: n=8)$ chronic obstructive pulmonary disease patients, $(b, h$ and $k: n=12 ; e: n=6) s m o k e r s$ and $(c, i$ and $\mathrm{l}: \mathrm{n}=12 ; \mathrm{f}: \mathrm{n}=4$ ) nonsmokers are shown. Cell nuclei were counterstained with either Mayer's haematoxylin (blue; a-i) or 4',6-diamidino-2-phenylindole (blue; $\mathrm{j}-\mathrm{l}$ ). For immunohistochemical analysis phospho-p38 MAPK expression was detected using 3,3'-diaminobenzadine (brown; a-i). For dual label immunofluorescence $(j-1)$ lung tissue neutrophils were identified using an Alexa-488 conjugated goat anti mouse secondary antibody (red; $j$ ) and phospho-p38 MAPK was detected using an Alexa 468 conjugated goat anti-rabbit secondary antibody (green; k). Composite images for dual label immunofluorescence are also shown (1). Phospho-p38 MAPK expression in alveolar macrophages (brown; a-c), sputum macrophages (brown; d-f) and small airway epithelial cells (brown; gi). Lung tissue neutrophils (red) expressing phospho-p38 MAPK (green; $j-1$ ). Magnification $\times 200$. Scale bars $=75 \mu \mathrm{m}$.

patients. Inhibition of p38 MAPK significantly reduced IFN- $\gamma$ release from isolated CD8 cells from COPD lungs. It is known that p38 MAPK signalling is involved in cytokine production from blood CD8 cells [25], and we now confirm a similar role for this pathway in lung CD8 cells. It has recently been demonstrated that the effect of glucocorticoids on IFN- $\gamma$ release from bronchoalveolar lavage lymphocytes is reduced in 

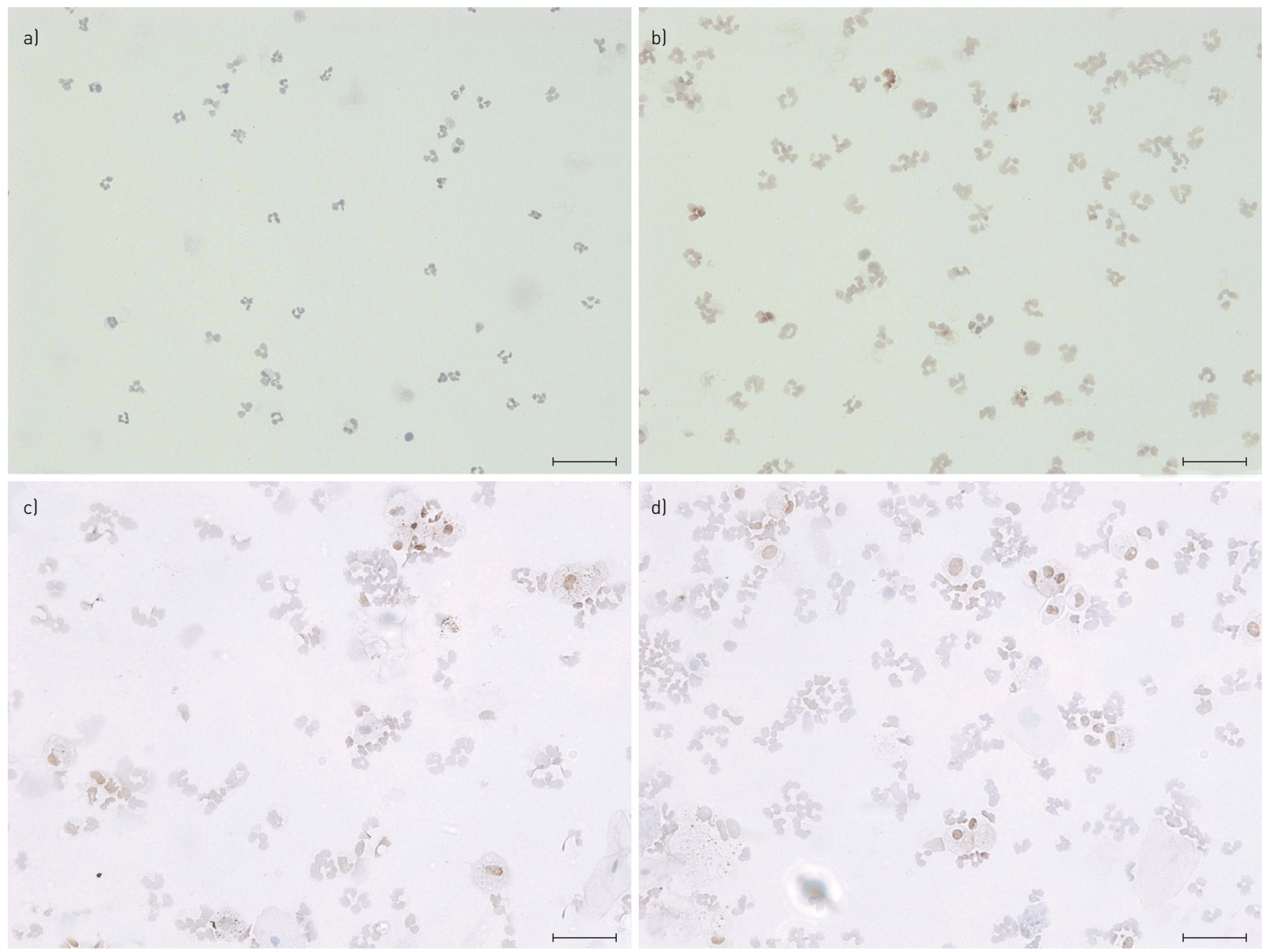

FIGURE 6 Representative images for the immunohistochemical detection of phosphorylated (phospho-)p38 mitogen-activated protein kinase (MAPK) in isolated chronic obstructive pulmonary disease (COPD) blood (a and b) and sputum (c and d) neutrophils. Cell nuclei were counterstained with Mayer's haematoxylin (blue). Phospho-p38 MAPK expression was detected using 3,3'-diaminobenzadine following direct immunohistochemistry (brown). a) Phosphop38 MAPK expression is absent in basal blood neutrophils. b) Phospho-p38 MAPK (brown) is induced in blood neutrophils following stimulation with $1 \mu \mathrm{g} \cdot \mathrm{mL}^{-1}$ lipopolysaccharide (LPS). Phospho-p38 MAPK is absent in c) basal and d) LPS-stimulated sputum neutrophils. Scale bars $=75 \mu \mathrm{m}$.

COPD patients compared with controls [26]. Furthermore, IFN- $\gamma$ causes glucocortiocoid insensitive cytokine production in COPD alveolar macrophages through signal transducers and activators of transcription (STAT)1 activation [27]. Inhibiting p38 MAPK signalling in lung CD8 cells can therefore target glucocorticoid insensitive mechanisms, such as IFN- $\gamma$ production from CD8 cells and, hence, subsequent STAT1-mediated cytokine production from macrophages.

Cigarette smoke induces the release of a variety of pro-inflammatory mediators from bronchial epithelial cells in vitro [28-31], implicating the epithelium in the pathogenesis of COPD. In the present study, the percentage of small airway epithelial cells positive for phospho-p38 MAPK was significantly higher in COPD lungs. Increased phospho-p38 MAPK expression in airway epithelial cells has also been found in severe asthma [32]. COPD and severe asthma are characterised by persistently increased levels of proinflammatory mediators in the airways $[33,34]$. The p38 MAPK pathway is activated by a range of inflammatory stimuli, and it appears that bronchial epithelial cells in both severe asthma and COPD respond to these stimuli by 338 MAPK activation.

Lung neutrophils were devoid of phospho-p38 MAPK immunoreactivity in all our patient groups, including smoking and nonsmoking controls. Stimulation of lung neutrophils with LPS did not induce phospho-p38 MAPK activation, indicating that the pro-inflammatory activity of lung neutrophils is not dependent on p38 MAPK signalling. This was confirmed by the lack of an effect of SB100 on 

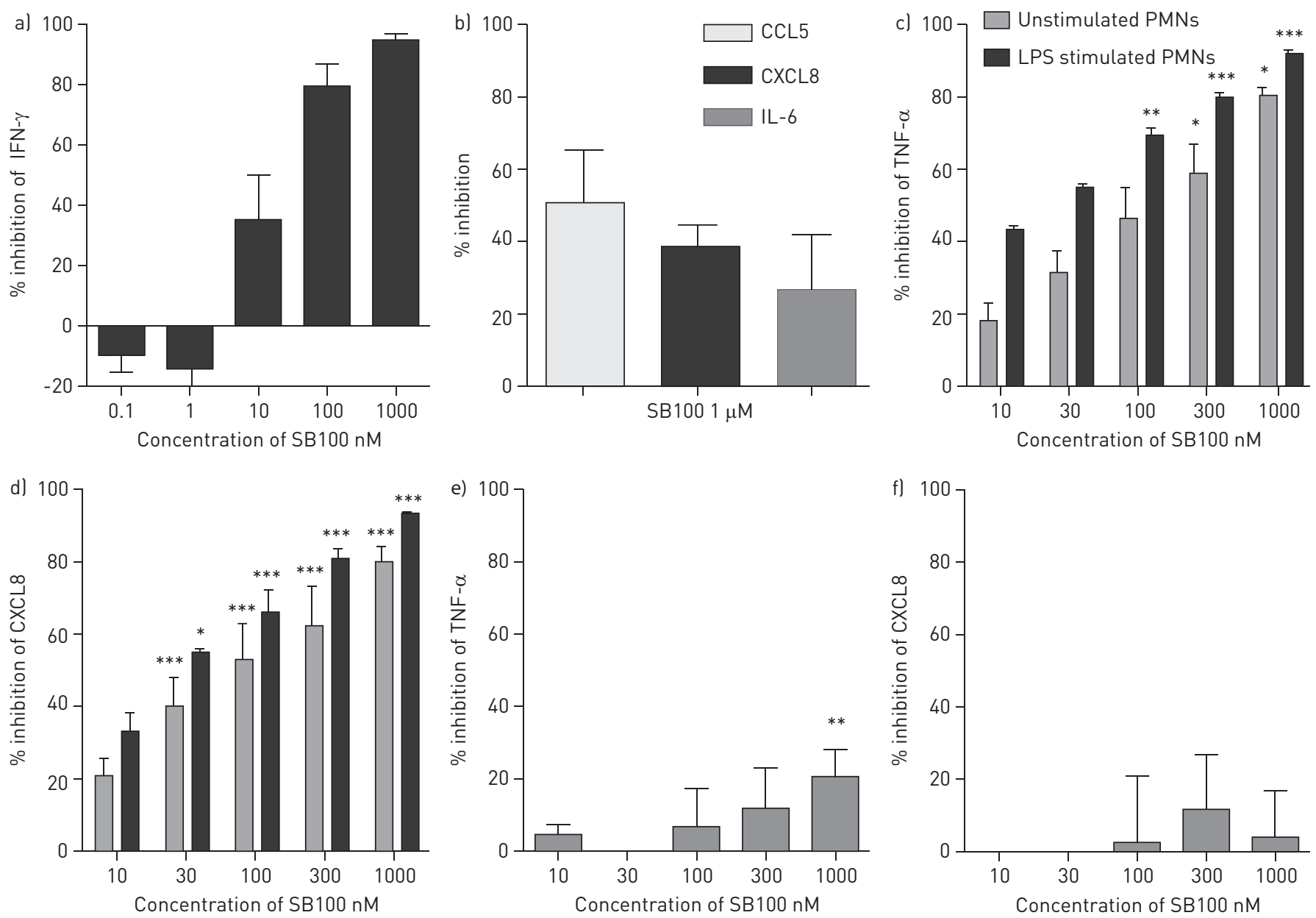

FIGURE 7 Effect of p38 mitogen-activated protein kinase (MAPK) inhibition in chronic obstructive pulmonary disease (COPD) cells. a) Inhibition of interferon (IFN)- $\gamma$ release from COPD lung CD8 cells $(\mathrm{n}=3)$ by SB100 $(0.1-1000 \mathrm{nM})$ following 24-h stimulation with interleukin (IL)-12 (10 ng $\left.\cdot \mathrm{mL}^{-1}\right)$ and $\mathrm{IL}-18$ $\left(10 \mathrm{ng} \cdot \mathrm{mL}^{-1}\right)$. b) Inhibition of chemokine (C-C motif) ligand 5 (CCL5), CXCL8 and IL-6 from epithelial cells isolated from COPD lung tissue ( $\mathrm{n}=3$ ) by SB100 $(1000 \mathrm{nM})$ following 24-h stimulation with polyinosinic:polycytidylic acid (poly I:C) $\left(10 \mu \mathrm{g} \cdot \mathrm{mL}^{-1}\right)$. Inhibition of c) tumour necrosis factor (TNF)- $\alpha$ and d) CXCL8 in stimulated and unstimulated isolated COPD blood neutrophils $(\mathrm{n}=7)$ following incubation with SB100 $(0.1-1000 \mathrm{nM})$ for 24 h. Inhibition of e) TNF- $\alpha$ and $f)$ CXCL8 release from sputum neutrophils $(n=7)$ following incubation with SB100 $(0.1-1000 \mathrm{nM})$ for 24 h. Data is presented as mean \pm SEM. A significant reduction in cytokine release following SB100 treatment is denoted by ${ }^{*}: \mathrm{p}<0.05 ;{ }^{* *}$ : $<<0.01$; and ${ }^{* * *}: \mathrm{p}<0.001$.

pro-inflammatory cytokine production from lung neutrophils. In contrast, phospho-p38 MAPK activation was observed in blood neutrophils, and cytokine production from these cells was inhibited by SB100. Our results suggest that SB100 inhibits both pre-formed and de novo synthesised cytokines in blood neutrophils, as similar inhibition was observed in both unstimulated and LPS-stimulated cells. Our results suggest that neutrophils leaving the bloodstream and entering the lung undergo phenotypic changes, altering the activity of intracellular signalling pathways required for important cell functions. Similarly, we have previously observed normal glucocorticoid receptor expression in blood neutrophils, but depleted glucocorticoid receptor expression in airway neutrophils [35]. These data highlight a potential pitfall of using blood neutrophils as a model for lung neutrophils, as there appear to be differences in the signalling mechanisms responsible for cytokine production.

Previous studies have demonstrated that p38 MAPK inhibition attenuates chemotaxis [36-39] and superoxide generation [40], in addition to pro-inflammatory mediator generation [41-43] in blood neutrophils. The lack of activated p38 MAPK observed in lung neutrophils makes it unlikely that p38 MAPK inhibition would have any effect on chemotaxis and superoxide generation in lung neutrophils. The altered phenotype of lung neutrophils, with reduced activation of p38 MAPK and expression of glucocorticoid receptor [35], suggests that specific anti-neutrophil therapies are needed to target this cell type in COPD, rather than broad anti-inflammatory drugs. 
In alveolar macrophages, we have previously shown that p38 MAPK activation is glucocorticoid resistant [11]. p38 MAPK inhibitors therefore target a glucocorticoid-resistant pathway that is activated within the lungs of COPD patients, and we show here that these drugs can suppress cytokine production from COPD lung lymphocytes and epithelial cells. There are synergistic interactions between p38 MAPK and glucocorticoids; both drugs used together cause a greater than additive inhibition of cytokine production from COPD alveolar macrophages [11]. There are molecular mechanisms that can explain such observations, such as glucocorticoid induced upregulation of MAPK phosphatase [44], which dephosphorylates p38 MAPK. Combination treatment with glucocorticoids and p38 MAPK inhibitors may also have synergistic effects on cytokine production from lymphocytes and epithelial cells.

We and others have reported no differences between COPD patients and controls for the effect of p38 inhibition on the release of inflammatory mediators from alveolar macrophages [9, 11]. We now report the effect of p38 inhibition in lung CD8 and epithelial cells from COPD patients, and it would be of interest to know if these effects differ from controls.

In conclusion, we show cell-specific activation of the p38 MAPK pathway in the lungs of COPD patients. p38 MAPK inhibitors suppress cytokine production from COPD lung lymphocytes and epithelial cells, but have no effect on lung neutrophils. These novel drugs therefore target some, but not all, of the inflammatory processes involved in COPD.

\section{References}

1 Hogg JC, Chu F, Utokaparch S, et al. The nature of small-airway obstruction in chronic obstructive pulmonary disease. N Engl J Med 2004; 350: 2645-2653.

2 Bourbeau J, Rouleau MY, Boucher S. Randomised controlled trial of inhaled corticosteroids in patients with chronic obstructive pulmonary disease. Thorax 1998; 53: 477-482.

3 Celli BR, Thomas NE, Anderson JA, et al. Effect of pharmacotherapy on rate of decline of lung function in chronic obstructive pulmonary disease: results from the TORCH study. Am J Respir Crit Care Med 2008; 178: 332-338.

4 Saklatvala J. The p38 MAP kinase pathway as a therapeutic target in inflammatory disease. Curr Opin Pharmacol 2004; 4: 372-377.

5 Chung KF. p38 mitogen-activated protein kinase pathways in asthma and COPD. Chest 2011; 139: 1470-1479.

6 Saccani S, Pantano S, Natoli G. p38-Dependent marking of inflammatory genes for increased NF-kappa B recruitment. Nat Immunol 2002; 3: 69-75.

7 Winzen R, Kracht M, Ritter B, et al. The p38 MAP kinase pathway signals for cytokine-induced mRNA stabilization via MAP kinase-activated protein kinase 2 and an AU-rich region-targeted mechanism. EMBO J 1999; 18: 4969-4980.

8 Birrell MA, Wong S, McCluskie K, et al. Second-generation inhibitors demonstrate the involvement of p38 mitogen-activated protein kinase in post-transcriptional modulation of inflammatory mediator production in human and rodent airways. J Pharmacol Exp Ther 2006; 316: 1318-1327.

9 Smith SJ, Fenwick PS, Nicholson AG, et al. Inhibitory effect of p38 mitogen-activated protein kinase inhibitors on cytokine release from human macrophages. Br J Pharmacol 2006; 149: 393-404.

10 Kent LM, Smyth LJ, Plumb J, et al. Inhibition of lipopolysaccharide-stimulated chronic obstructive pulmonary disease macrophage inflammatory gene expression by dexamethasone and the p38 mitogen-activated protein kinase inhibitor $\quad N$-cyano- $N$-(2-\{[8-(2,6-difluorophenyl)-4-(4-fluoro-2-methylphenyl)-7-oxo-7,8-dihydropyrido[2,3-d] pyrimidin-2-yl]amino\}ethyl)guanidine (SB706504). J Pharmacol Exp Ther 2009; 328: 458-468.

11 Armstrong J, Harbron C, Lea S, et al. Synergistic effects of p38 mitogen-activated protein kinase inhibition with a corticosteroid in alveolar macrophages from patients with chronic obstructive pulmonary disease. J Pharmacol Exp Ther 2011; 338: 732-740.

12 Singh D, Smyth L, Borrill Z, et al. A randomized, placebo-controlled study of the effects of the p38 MAPK inhibitor SB-681323 on blood biomarkers of inflammation in COPD patients. J Clin Pharmacol 2010; 50: 94-100.

13 Lomas DA, Lipson DA, Miller BE, et al. An oral inhibitor of p38 MAP kinase reduces plasma fibrinogen in patients with chronic obstructive pulmonary disease. J Clin Pharmacol 2012; 52: 416-424.

14 Agusti A, MacNee W, Donaldson K, et al. Hypothesis: does COPD have an autoimmune component? Thorax 2003; 58: 832-834.

15 Renda T, Baraldo S, Pelaia G, et al. Increased activation of p38 MAPK in COPD. Eur Respir J 2008; 31: 62-69. Zarubin T, Han J. Activation and signaling of the p38 MAP kinase pathway. Cell Res 2005; 15: 11-18.

Polzer K, Soleiman A, Baum W, et al. Selective p38MAPK isoform expression and activation in antineutrophil cytoplasmatic antibody-associated crescentic glomerulonephritis: role of p38MAPKalpha. Ann Rheum Dis 2008; 67: 602-608.

18 Global Initiative for Chronic Obstructive Lung Disease. GOLD Spirometry Guide. GOLD, 2010. www.goldcopd. org/other-resources-gold-spirometry-guide.html

19 Plumb J, Smyth LJ, Adams HR, et al. Increased T-regulatory cells within lymphocyte follicles in moderate COPD. Eur Respir J 2009; 34: 89-94.

20 Nick JA, Avdi NJ, Young SK, et al. Selective activation and functional significance of p38alpha mitogen-activated protein kinase in lipopolysaccharide-stimulated neutrophils. J Clin Invest 1999; 103: 851-858.

21 Baines KJ, Simpson JL, Scott RJ, et al. Immune responses of airway neutrophils are impaired in asthma. Exp Lung Res 2009; 35: 554-569.

22 Mercado N, To Y, Kobayashi Y, et al. p38 mitogen-activated protein kinase-gamma inhibition by long-acting beta2 adrenergic agonists reversed steroid insensitivity in severe asthma. Mol Pharmacol 2011; 80: 1128-1135.

23 van der Strate BW, Postma DS, Brandsma CA, et al. Cigarette smoke-induced emphysema: a role for the B cell? Am J Respir Crit Care Med 2006; 173: 751-758. 

chronic obstructive pulmonary disease. Clin Exp Immunol 2009; 155: 466-475.

25 Merritt C, Enslen H, Diehl N, et al. Activation of p38 mitogen-activated protein kinase in vivo selectively induces apoptosis of CD8(+) but not CD4(+) T cells. Mol Cell Biol 2000; 20: 936-946.

26 Kaur M, Smyth LJ, Cadden P, et al. T lymphocyte insensitivity to corticosteroids in chronic obstructive pulmonary disease. Respir Res 2012; 13: 20.

27 Southworth T, Metryka A, Lea S, et al. IFN- $\gamma$ synergistically enhances LPS signalling in alveolar macrophages from COPD patients and controls by corticosteroid-resistant STAT1 activation. Br J Pharmacol 2012; 166: 2070-2083.

28 Beisswenger C, Platz J, Seifart C, et al. Exposure of differentiated airway epithelial cells to volatile smoke in vitro. Respiration 2004; 71: 402-409.

29 Hellermann GR, Nagy SB, Kong X, et al. Mechanism of cigarette smoke condensate-induced acute inflammatory response in human bronchial epithelial cells. Respir Res 2002; 3: 22.

30 Mio T, Romberger DJ, Thompson AB, et al. Cigarette smoke induces interleukin-8 release from human bronchial epithelial cells. Am J Respir Crit Care Med 1997; 155: 1770-1776.

31 Takizawa H, Tanaka M, Takami K, et al. Increased expression of transforming growth factor-betal in small airway epithelium from tobacco smokers and patients with chronic obstructive pulmonary disease (COPD). Am J Respir Crit Care Med 2001; 163: 1476-1483.

32 Liu W, Liang Q, Balzar S, et al. Cell-specific activation profile of extracellular signal-regulated kinase 1/2, Jun Nterminal kinase, and p38 mitogen-activated protein kinases in asthmatic airways. J Allergy Clin Immunol 2008; 121: 893-902.

33 Miller M, Cho JY, Pham A, et al. Persistent airway inflammation and emphysema progression on CT scan in exsmokers observed for 4 years. Chest 2011; 139: 1380-1387.

34 Wenzel SE, Szefler SJ, Leung DY, et al. Bronchoscopic evaluation of severe asthma. Persistent inflammation associated with high dose glucocorticoids. Am J Respir Crit Care Med 1997; 156: 737-743.

35 Plumb J, Gaffey K, Kane B, et al. Reduced glucocorticoid receptor expression and function in airway neutrophils. Int Immunopharmacol 2012; 12: 26-33.

36 Fujita T, Zawawi KH, Kurihara H, et al. CD38 cleavage in fMLP- and IL-8-induced chemotaxis is dependent on p38 MAP kinase but independent of p44/42 MAP kinase. Cell Signal 2005; 17: 167-175.

37 Nick JA, Avdi NJ, Young SK, et al. Common and distinct intracellular signaling pathways in human neutrophils utilized by platelet activating factor and FMLP. J Clin Invest 1997; 99: 975-986.

38 Nick JA, Young SK, Brown KK, et al. Role of p38 mitogen-activated protein kinase in a murine model of pulmonary inflammation. J Immunol 2000; 164: 2151-2159.

39 Cara DC, Kaur J, Forster M, et al. Role of p38 mitogen-activated protein kinase in chemokine-induced emigration and chemotaxis in vivo. J Immunol 2001; 167: 6552-6558.

40 Suzuki K, Hino M, Kutsuna H, et al. Selective activation of p38 mitogen-activated protein kinase cascade in human neutrophils stimulated by IL-1beta. J Immunol 2001; 167: 5940-5947.

41 Zu YL, Qi J, Gilchrist A, et al. p38 mitogen-activated protein kinase activation is required for human neutrophil function triggered by TNF-alpha or FMLP stimulation. J Immunol 1998; 160: 1982-1989.

42 Coxon PY, Rane MJ, Uriarte S, et al. MAPK-activated protein kinase-2 participates in p38 MAPK-dependent and ERK-dependent functions in human neutrophils. Cell Signal 2003; 15: 993-1001.

43 Ribeiro FP, Furlaneto CJ, Hatanaka E, et al. mRNA expression and release of interleukin- 8 induced by serum amyloid A in neutrophils and monocytes. Mediators Inflamm 2003; 12: 173-178.

44 Abraham SM, Lawrence T, Kleiman A, et al. Antiinflammatory effects of dexamethasone are partly dependent on induction of dual specificity phosphatase 1. J Exp Med 2006; 203: 1883-1889. 